\title{
To Adhere or Not to Adhere: An Important Decision
}

\author{
By Jack M. Gorman, MD
}

My former Mount Sinai colleague Eyal Shemesh, MD, guest editor of this month's CNS Spectrums, kindly asked me to serve as a discussant for a seminar he had proposed for the 2005 meeting of the American Psychiatric Association (APA). The idea for the symposium grew out of an observation that Dr. Shemesh made while reading an article in a prominent cardiology journal. The article identified post-myocardial infarction (MI) patients who appeared nonresponsive to aspirin therapy, and it speculated on a variety of reasons that may explain aspirin "resistance" in patients. However, Dr. Shemesh noticed that one seemingly obvious reasonlack of adherence-was not mentioned in this article. With his close colleague, cardiologist Gad Cotter, MD, Dr. Shemesh found that failure to respond to aspirin was caused by a postMI patient's non-compliance to the prescribed treatment regimen. Dr. Shemesh has also begun studies indicating that, thus far, traumatic stress is associated with lack of adherence in these patients and that a relatively simple psychosocial intervention can address this issue and improve overall outcomes.

Adherence is an increasingly important topic for all health professionals, but it is still underrecognized as an important factor in whether patients with a variety of diseases will improve. When protease inhibitors were first introduced for the treatment of human immunodeficiency virus (HIV) infection, it was realized that adherence would play a major role in outcomes. This is because although modern regimens for HIV infection are highly effective, the virus can become resistant to many medications if they are not taken on schedule. These drug regimens, however, can be demanding and it quickly became apparent that failure to adhereadherence defined as taking every pill every time-could lead to viral resistance and drastic consequences. Hence, the HIV research field became deeply involved in factors that contribute to lack of medication adherence.

Other areas of medicine have been slower to delve into this area, but there is growing appreciation that a leading cause of treatment failure is non-adherence. A recent lecturer at Mount Sinai commented that only $\sim 30 \%$ of glaucoma patients regularly take their eye drops, a staggering observation. Given that the consequences of such non-adherence could be blindness, adherence would seem relatively simple. It would also seem that figuring out how to improve patients' track records of taking medications that physicians can already prescribe may be as important a research topic as developing new medications themselves.

Physicians are often dismissive of and even angry at patients who do not take their medication or follow other medical recommendations. I remember in medical school, however, that several of my classmates converted to positive PPDs and were advised to take isoniazid on a daily basis for 1 year. Most did so...for about a week. Then, they complained that it was a big pill, that they did not feel ill, and other excuses. Not much different from the typical patient. There is no evidence that physicians are better than any other segment of the population in taking their prescribed medications. 
An important aspect of the research described in this month's articles is that psychiatric symptoms, such as depression and anxiety, may be key factors in non-adherence. At the APA symposium, Dr. Cotter informed the audience that the long-held belief that patients with hypertension who do not regularly take their antihypertensive medications do so because they do not feel ill is false. According to Dr. Cotter, more recent studies have shown that comorbid psychiatric illness is the leading cause of non-adherence to antihypertensive medication regimens. If this turns out to be true, one obvious solution might be to identify and treat psychiatric illness in those with other medical problems more vigorously than is done currently. Surprisingly, few studies have examined whether successful treatment of comorbid illnesses will improve adherence. This is clearly an area ripe for future investigation.

Before closing this month's column, I would like to turn your attention to the case presentation and discussion by Dan J. Stein, MD, PhD. Once again, this column is both informative and elegant. Dr. Stein is one of the leading experts in combining neurobiological information with psychosocial insights. His bi-monthly column continues to be a highlight for CNS Spectrums. CNS

\section{CALL FOR PAPERS}

CNS Spectrums is accepting submissions of case reports, review articles, and original research on a variety of neuroscientific and clinical neuropsychiatric topics. Examples of topics include:

\section{- Clinical psychiatry}

- Neurology and neuropsychiatry in a clinical setting addressing spectrum disorders

- Applications of psychopharmacology and pharmacokinetics across the neuropsychiatric spectrum

Especially encouraged are papers covering comorbidities in neurologic disorders (eg, epilepsy with schizophrenia). Other crossover manuscripts geared to deepening the clinician's understanding of neuropsychiatric disorders and treatments will be given highest priority. (Please see "Author Guidelines" on page 73 for additional information or at www.cnsspectrums.com).

CNS Spectrums is the largest circulation Index Medicus publication with a circulation of 48,000 neurologists and psychiatrists.

Submissions should be sent to Jack M. Gorman, MD, Editor (In Europe, to Joseph Zohar, MD, International Editor), CNS Spectrums c/o MBL Communications, 333 Hudson Street, 7th Floor, NewYork, NY 10013.

\title{
CME1 \\ Available in March \\ CME-ACCREDITED SYMPOSIUM MONOGRAPH SUPPLEMENT
}

\author{
An expert review of clinical challenges in psychiatry
}

\section{Incorporating Pharmacogenetics Into Clinical Practice: The Reality of a New Tool in Psychiatry}

\author{
Moderator: \\ David A. Mrazek, MD \\ Discussants: Jordan W. Smoller, MD, ScD, and Jose de Leon, MD \\ Section Editor: David L. Ginsberg, MD
}

Supported through an unrestricted educational grant from Roche 